\title{
SIMULATION EVALUATION OF A LOW-ALTITUDE HELICOPTER FLIGHT GUIDANCE SYSTEM
ADAPTED FOR A HELMET-MOUNTED DISPLAY
}

\author{
Harry N. Swenson", Richard E. Zelenka*, \\ Gordon H. Hardyt, and Munro G. Dearing' \\ NASA-Ames Research Center \\ Moffett Field, CA 94035
}

\section{Abstract}

A computer aiding concept for low-altitude helicopter flight has been developed and evaluated in a real-time piloted simulation. The concept included an optimal control trajectory-generation algorithm based upon dynamic programming, and a helmet-mounted display (HMD) presentation of a pathway-in-the-sky, a phantom aircraft, and flight-path vector/predictor guidance symbology. The trajectory-generation algorithm uses knowledge of the global mission requirements, a digital terrain map, aircraft performance capabilities and advanced navigation information to determine a trajectory between mission waypoints that seeks valleys to minimize threat exposure. The pilot evaluation was conducted at the NASA-Ames Research Center moving base Vertical Motion Simulator (VMS) by pilots representing NASA, the U.S. Army, Air Force, and helicopter industry. The pilots manually tracked the trajectory generated by the algorithm utilizing the HMD symbology. The pilots were able to satisfactorily perform the tracking tasks while maintaining a high degree of awareness of the outside world.

\section{Introduction}

Helicopters that operate in threat areas have a need for low-altitude, maneuvering-penetration capability for nighttime and adverse weather conditions. Currently this low-altitude penetration is accomplished through terrain-following (TF) systems by using a combination of technologies such as multimode radar (MMR) systems, forward looking-infrared (FLIR) and night-vision goggles (NVG). TF systems were initially developed for fixedwinged tactical and strategic aircraft such as the FB-111 and B-1B. The TF systems have also been developed for combat search and rescue aircraft such as the $\mathrm{CH}-53$ PAVE LOW III and the HH-60 helicopters, and are currently part of the Army's Special Operations Forces (SOF) helicopters. 1 The TF systems generate vertical commands which are displayed on a flight director for manual flight or sent to the flight-control system for automatic flight. These systems do not generate commands for lateral maneuvering, are limited to line-of-

- Research Engineer

$\dagger$ Research Test Pilot

This paper is declared a work of the U.S. Government and therefore is in the public domain sight maneuvering and do not provide information to the pilot to allow him to make strategic decisions that could give better terrain masking.

Recently the Air Force has sponsored research to extend TF capability for high-performance aircraft to include lateral maneuvering by taking advantage of onboard digital terrain data.2-5 The work concentrated on the development of four potential algorithms, each of which is based on minimizing a quadratic cost functional, defined to reflect the degree of vulnerability. This extended capability is commonly referred to as Terrain Following/Terrain Avoidance (TF/TA) in the literature.

Within the last few years there has been considerable work at NASA and elsewhere in applying these algorithms to rotorcraft. 6-8 The NASA research has concentrated on incorporating these algorithms into an operationally acceptable system, referred to as the Computer Aiding for Low-Altitude Helicopter Flight (CALAHF) guidance system. Several piloted simulations of the CALAHF guidance system, have been conducted. The first two were dedicated to the development of the system and pilot interface issues. 9 The third simulation was an operational evaluation of these guidance and display concepts to determine tracking performance for various flight and environmental conditions, and pilot situational awareness. 10

The very favorable pilot feedback and response from the operational simulation described in Reference (10) has led to a joint NASA and U.S. Army flight test of the CALAHF flight guidance system. The NASA developed system will be flown on the U.S. Army Avionics Research and Development Activity's UH-60 STAR (Şystem Iestbed for Ávionics Besearch) research helicopter. The purpose of this paper is 1) to describe the flight guidance and display system to be installed in the UH-60 STAR, and 2) describe an operational evaluation of the flight test modified system on the NASA-Ames Vertical Motion Simulator (VMS). This paper describes the overall system, which includes: 1) the trajectory-generation algorithm, 2) the trajectory coupler, and 3) the displayed information. The simulation, test procedures and performance results are then presented. 


\section{System Description}

Shown in Figure 1 is a functional block diagram of the computer-aiding for low-altitude helicopter flight system. The three fundamental components are: 1) the Dynapath trajectory-generation algorithm; 2) the trajectory coupler; and 3) the displayed information This system has to be integrated with the pilot, helicopter, and the aircraft sensors. The trajectorygeneration algorithm, the trajectory coupler, and the displayed information are discussed below.

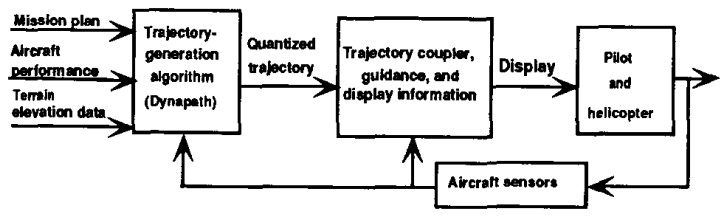

Figure 1. System block diagram.

\section{Irajectory Generation Algorithm}

The trajectory-generation algorithm (Figure (1)), also known as Dynapath, was originally developed for the U.S. Air Force. 11 The goal of the Air Force research was the development of a TF/TA guidance algorithm for automatic tactical aircraft operations. Significant modifications have been made to this guidance algorithm in adapting it for manual rotorcraft operations. 9,10

Dynapath is a valley-seeking trajectory-generating algorithm based on a forward-chaining dynamicprogramming technique. In-depth descriptions can be found in References (11) and (12), so it will be treated only briefly here. The algorithm uses two types of inputs. The first, characterized as mission dependent information, includes mission waypoints for defining a global trajectory to be flown, and Defense Mapping Agency (DMA) digital terrain elevation data (DTED) of the area in which the mission is to be accomplished. The second kind of input consists of pilot-comfort and aircraft-dependent parameters: maximum bank angle commands, maximum climb and dive angles, maximum pull up and push over load factor, set-clearance altitude (desired trajectory altitude above the ground) along with sensed aircraft state information.

Dynapath uses a decoupled procedure in which the lateral and vertical trajectory solutions are determined independently to obtain an optimal trajectory. In this decoupled procedure, the lateral ground track is first determined by assuming that the aircraft can fly perfectly at the vertical set-clearance altitude (desired altitude above ground level). The vertical trajectory is then calculated using aircraft normal load factor and flight path angle as maneuver constraints to maintain the aircraft at or slightly above the vertical set clearance determined from the digital terrain map and the lateral ground track.
The lateral path is calculated using a tree structure of possible two-dimensional trajectories by using discretized variation in aircraft bank angle. Assuming constant speed and coordinated flight, each discrete bank value produces a possible path which in combination forms a tree of possible paths (Figure (2)). In this implementation, the bank angle control has five discrete values that are used for the trajectory calculation. They are $\pm 100 \%$ maximum bank angle for large control, $\pm 33 \%$ maximum bank angle for fine control and $0^{\circ}$ bank angle. At any node point only three bankangle control values can be used: the control used in arriving at the node point and the control values to either side. At each successive node of the tree, the aircraft position and heading are stored along with the cumulative cost to each node. A grid is superimposed upon the tree structure with boundaries defined by the maximum lateral deviation and length of optimization. The purpose of the grid is to allow pruning of the tree to keep the number of possible tree branches at a reasonable level. The size and number of grid elements or "cells" were determined experimentally. For a 30-sec patch the number of cell divisions is 20 longitudinally along the patch and 20 laterally across the patch. Pruning the tree after three to four levels of branching gave the best mix of branch generation and computational speed based upon results from non-realtime computer simulations. 13 Pruning is accomplished by comparing each node within a cell that is heading in approximately the same direction and choosing the one with the lowest cost to continue the branch propagation. Pruning is also performed on branches that travel outside the grid or in a direction that causes significant path reversals, and is done at each node generation. After the tree structure of possible paths has been propagated through the entire patch length, the cumulative cost $(\mathbf{J})$ of all surviving branches are compared, and the path with the lowest cost is selected as the optimal trajectory.

The cost function $\mathbf{J}$ is the performance measure used to determine the optimal trajectory:

$$
J=\sum_{i=1}^{30} H_{i}^{2}+f(D) \omega D_{i}^{2}+\alpha\left(\Delta \Psi_{i}\right)^{2}
$$

where

$$
\begin{aligned}
& \boldsymbol{H}_{i} \quad=\text { altitude above mean sea level at node } \mathrm{i}(\mathrm{ft} \text { ) } \\
& D_{i} \quad=\text { lateral distance from reference path at } \\
& \text { node } i \text { (ft) } \\
& \omega=\text { TF/ TA ratio } \\
& f(D)=\text { dead band on lateral deviation cost i.e. } \\
& \text { if }|\mathrm{D}|<\delta \text { then } f(D)=0 \text { where } \delta \text { is a } \\
& \text { meaningful distance, else } f(D)=1 \text {, } \\
& \text { and } D_{1}=\left(\left|D_{i}\right|-|\delta|\right) \\
& \Delta \Psi_{1}=\text { the difference between the reference heading } \\
& \text { and the commanded heading at node } i \text { (deg) } \\
& \boldsymbol{\alpha}=\text { heading weight }
\end{aligned}
$$




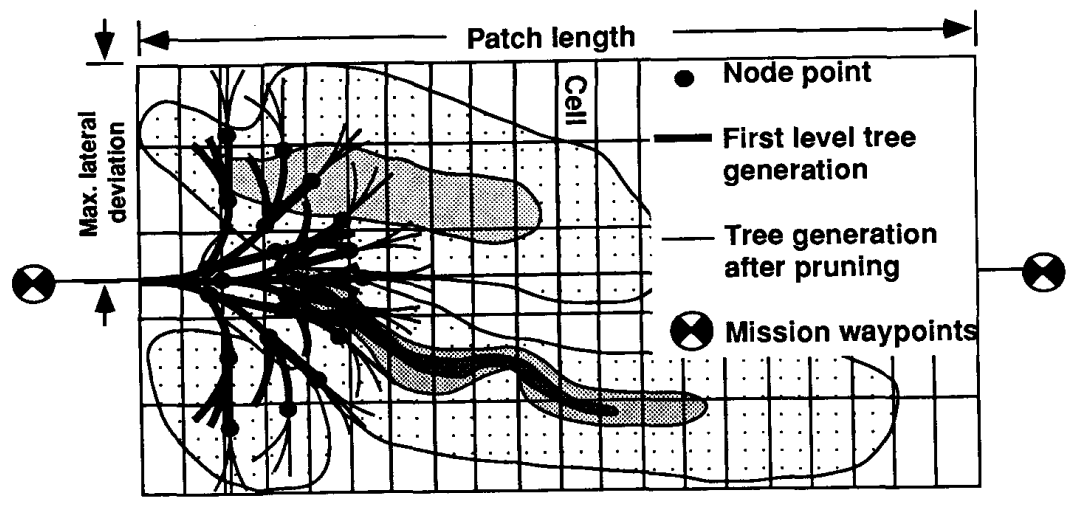

Figure 2. Dynapath tree generation.

The rationale for this particular cost functional is given in Reference (9), but a brief description is warranted here. The fundamental parameters in this performance measure are the terms representing altitude $\mathrm{H}$ and reference-path deviation $D$. The cost-functional, when driven by these two terms, allows lateral maneuvering to seek lower altitude terrain by the cost reduction from $\mathrm{H}$; excessive deviation from the reference path is controlled by increasing cost due to $D$. The TF/TA ratio $\omega$ allows blending of these two terms to obtain a desired balance between vertical and horizontal maneuvering. The $f(D)$ and $\alpha\left(\Delta \Psi_{i}\right)$ terms were added to reduce undesirable oscillations in the trajectory about the nominal path within a patch that are caused by the bank-angle quantization. The $f(D)$ eliminates the need for precise following of the nominal, or reference path, and the $\alpha\left(\Delta \Psi_{i}\right)$ term provides a penalty for changing the heading from that given by the reference path. These two terms were added as a result of experience gained in piloted simulations to make the trajectory-generation algorithm emulate pilot control strategies for low-altitude maneuvering flight. 9

The trajectory-generation algorithm, as defined above, is designed to compute guidance for a patch which is the area in front of the aircraft's present location. The patch width is the maximum lateral deviation, and the length is the flight preview distance. Both are input parameters selected by the user. The algorithm is computationally intensive and using representative values for patch length $(=30 \mathrm{sec})$ and maximum lateral deviation $(\approx 1 \mathrm{~km})$ the computational cycle is approximately 4 to $5 \mathrm{sec}$ for a modern ( 1 to 2 mip) flight computer. Several methods to update and propagate the algorithm were developed and evaluated during piloted simulations.9,10 The algorithm is initialized to a predicted location of the aircraft one computational cycle from its current position based upon its current position, velocity and computational cycle time. The trajectory-generation algorithm then calculates the first patch. Once the first patch is calculated the algorithm queries the aircraft for its current location to determine whether the aircraft has traveled into the first patch. Upon entering the patch, the algorithm updates itself in a bootstrapping fashion by first selecting a point into the patch corresponding to the time required for the computational cycle time (4 to 5 sec) plus the time corresponding to the length of the pilot's pathway-in-the-sky display (7 to $10 \mathrm{sec}$ ). Next, using this point, the second patch is calculated. Finally, each subsequent patch is initiated using the trajectory point from the preceding patch corresponding to the end of the pilot's pathway-in-the-sky display, thus freezing the initial segment of the trajectory. In this way, the algorithm update is imperceptible to the pilot.

\section{Irajectory Coupler}

After the Dynapath algorithm produces its optimal trajectory it is passed to the trajectory coupler. The trajectory is represented by 30 discrete instances of commanded aircraft-inertial state (position, velocity and acceleration) at $1 \mathrm{sec}$ intervals. Also stored are commanded bank angles, headings and vertical flightpath angles. The trajectory coupler, converts the quantized commanded trajectory into a trajectory command that is designed to work synchronously with the pilot displays at a minimum of $20 \mathrm{~Hz}$, thus not imposing any time-delay that is perceptible to the pilot This is accomplished by interpolating within the trajectory to determine the instantaneous position of the trajectory points to be presented on the pilots head-updisplay.

\section{Displayed Information}

The guidance and control information is displayed to the pilot on a helmet mounted display (HMD) in the format shown in Figure (3). The display device used was a Honeywell Integrated Helmet and Display Sighting System (IHADSS) This is a change from the heads-up display (HUD) used in the earlier concept development and operational evaluation described in References (9) and (10). The change was based upon a U.S. Army request to use a helmet mounted display. The HMD format is a mixture of screen, body, and inertially 


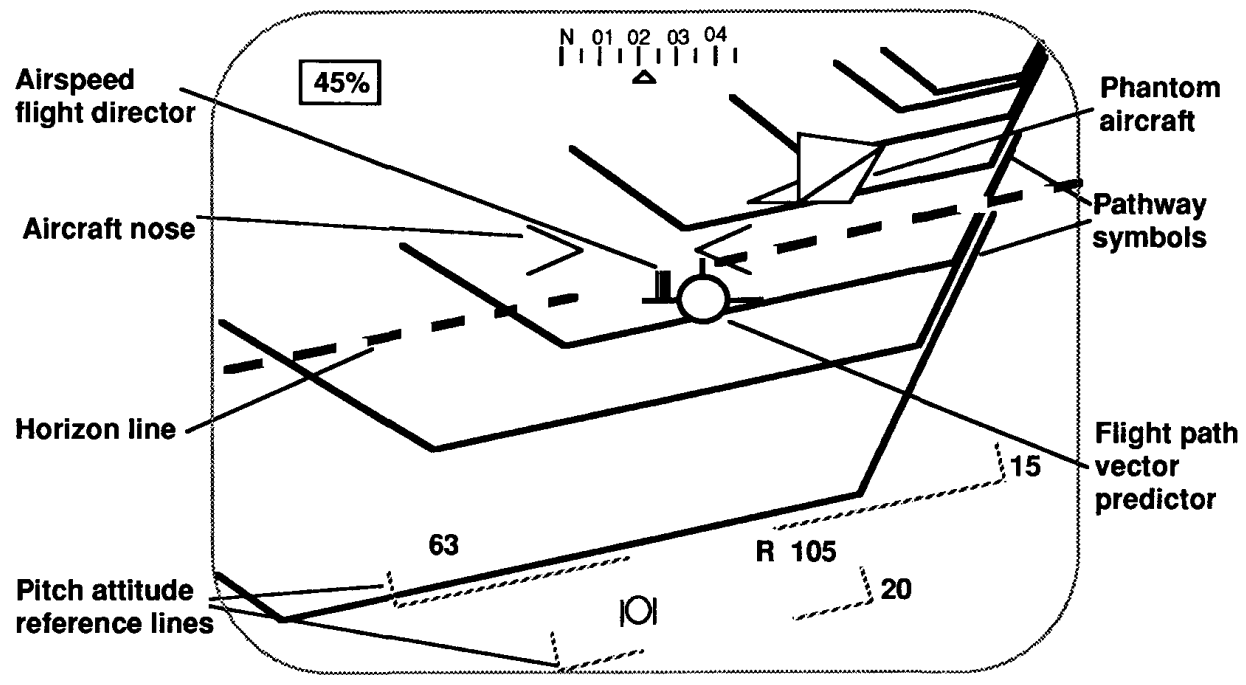

Figure 3. HMD format.

referenced symbols. The screen referenced symbols include; a heading tape $\left(021^{\circ}\right)$, engine torque ( $\left.45 \%\right)$, airspeed (63 kts), radar altitude ( $105 \mathrm{ft}$ ), and ball and slip indicator. The body referenced symbols are the aircraft nose ( $><$ ), and the flight-path vector/predictor. All remaining symbols are inertially referenced. The positioning of these symbols depends on aircraft attitude, and pilot head position. The pitch attitude reference lines are displayed at $5^{\circ}$ increments with the first lines at $\pm 15^{\circ}$, to avoid unnecessary clutter around the central display area. The horizon line will remain true to the actual horizon for all aircraft attitudes and pilot head angles, with the exception of head roll due to an artifact of the IHADSS head tracker which does not provide the signal. The primary situational information is presented to the pilot with an inertially stabilized flightpath vector/predictor symbol, represented by the circular aircraft icon with attached airspeed flight director tape. The logic that drives these symbols will be discussed later. The situational information presented on the HMD in Figure (3) indicates the pilot is turning right with a slight descent as indicated by the flight-path vector/predictor below the horizon, and is looking approximately along the longitudinal axis of the aircraft as indicated by the position of the aircraft nose symbol.

The Dynapath trajectory information on the HMD is given by the pathway-in-the-sky and phantom aircraft. The pathway symbols represent a three-dimensional perspective of the inertial position and heading of the discretized Dynapath trajectory. The phantom aircraft is displayed on the HMD as a delta-wing aircraft. The phantom aircraft represents the instantaneous position along the Dynapath trajectory that is 3,4 or $5 \mathrm{sec}$ ahead of the pilot's aircraft depending upon test configuration. The phantom aircraft attitude is also derived from the Dynapath trajectory, using the flight-path angle, bank angle, and heading as pitch, roll, and yaw respectively. By positioning the flight-path vector symbol on the phantom aircraft, the pilot will track the desired trajectory, this technique is referred to as pursuit tracking ${ }^{14}$. In Figure (3), the HMD symbols are presenting a climbing right turn.

The pathway is $100 \mathrm{ft}$ (roughly two rotor diameters) wide at the bottom and parallel to the horizon with vertical projections that are canted at a $45^{\circ}$ angle; the width at the top is $200 \mathrm{ft}$. The depth of the path is $50 \mathrm{ft}$ below the intended trajectory; thus when flying a level straight-line commanded path, the pilots used the analogy of traveling in a full irrigation canal for describing the pathway symbols. The pathway was varied from 7 to 20 lines as an experimental variable, Figure (3) shows the baseline configuration of 7 lines.

The pathway represents the actual Dynapath trajectory on the HMD. Thus if the trajectory is not visible in the field-of-view (FOV) of the HMD $\left( \pm 15^{\circ}\right.$ vertically and $\pm 20^{\circ}$ horizontally), then the pathway likewise is not displayed. However, if the phantom aircraft position occurs outside the FOV, the symbol is positioned on the edge of the HMD closest to the true position so that the pilot will know the direction to the path even though he can't see it. At this point the positioning of the flight-path vector/predictor symbol relative to the phantom aircraft will remain true. The relative position between the two symbols is the error term for pilot tracking and no longer situational data. This change in mode is indicated to the pilot by blinking the vector/predictor symbol at a rate of once per second. The pilot also has the option to reposition his head in the direction of the pathway, at which time it will be displayed.

The primary guidance information to the pilot is provided by the relative positions of the phantom aircraft symbol and the flight-path vector/predictor symbol. The vertical flight-path vector/predictor $\gamma_{v p}$ is driven by 


$$
\gamma_{v_{\mathrm{p}}}=\gamma_{v}+\frac{\tau_{\mathrm{H}} s}{\tau_{\mathrm{H}} s+1}\left(\mathrm{~K}_{\delta_{\mathrm{e}}} \delta_{\mathrm{c}}+\theta\right)
$$

\section{where}

$$
\begin{array}{ll}
\boldsymbol{\gamma}_{\mathbf{v}} & =\text { vertical flight }- \text { path angle }(\mathrm{rad}) \\
\boldsymbol{\tau}_{\mathbf{H}} & =\text { aircraft heave time }- \text { constant }(3 \mathrm{sec}) \\
\boldsymbol{s} & =\text { Laplace transform variable } \\
\mathbf{K}_{\mathbf{\delta}_{\mathbf{c}}} & =\text { collective input gain }(.095 \mathrm{rad} / \mathrm{in}) \\
\boldsymbol{\delta}_{\mathbf{c}} & =\text { collective stick input (inches) } \\
\boldsymbol{\theta} & =\text { pitch attitude }(\mathrm{rad})
\end{array}
$$

The horizontal flight-path vector/predictor $\gamma_{H p}$ is driven by

$$
\gamma_{H_{p}}=\gamma_{H}+\frac{g \tan (\phi) T_{p}}{2 V}
$$

where

$$
\begin{array}{ll}
\boldsymbol{\gamma}_{\mathbf{H}} & =\text { horizontal flight }- \text { path vector }(\mathrm{rad}) \\
\boldsymbol{g} & =\text { acceleration due to gravity }\left(\mathrm{ft} / \mathrm{sec}^{2}\right) \\
\boldsymbol{\phi} & =\text { aircraft bank angle }(\mathrm{rad}) \\
\boldsymbol{T}_{\boldsymbol{p}} & =\text { prediction time }(3,4, \text { or } 5 \mathrm{sec}) \\
\boldsymbol{V} & =\text { helicopter velocity }(\mathrm{ft} / \mathrm{sec})
\end{array}
$$

The airspeed flight director tape $\varepsilon_{v}$ is driven by

$$
\varepsilon_{v} \quad=K_{\varepsilon}\left(V_{\varepsilon}+\frac{\tau_{\theta} s}{\tau_{\theta} s+1}\left(K_{\theta} \theta\right)\right)
$$

where

$$
\begin{array}{ll}
\boldsymbol{K}_{\boldsymbol{\varepsilon}} & =\text { display error gain }(0.075 \mathrm{rad} /(\mathrm{ft} / \mathrm{sec})) \\
\boldsymbol{V}_{\boldsymbol{\varepsilon}} & =\text { velocity error (desired }- \text { actual })(\mathrm{ft} / \mathrm{sec}) \\
\boldsymbol{\tau}_{\boldsymbol{\theta}} & =\text { speed time }- \text { constant }(25 \mathrm{sec}) \\
\mathbf{K}_{\boldsymbol{\theta}} & =\text { filter gain }(5.62 \mathrm{rad} /(\mathrm{ft} / \mathrm{sec}))
\end{array}
$$

The vertical predictive term is compensation for the heave-time of the aircraft. Since the simulated helicopter's flight control system produces essentially vertical velocity in response to collective input, the collective feedback gives the pilot an immediate feedback of his control actions to the aircraft vertical flight-path angle. Therefore, the pilot can position the flight-path symbol vertically without waiting for the aircraft to respond. The horizontal prediction term projects along path curvature to determine where the aircraft will be $T_{p}$ sec from now based upon the aircraft's current bank angle and velocity. Therefore, the pilot can position the flight-path vector/predictor upon the phantom aircraft with little difficulty. The airspeed flight director is a tape that grows and shrinks in response to airspeed error and longitudinal acceleration derived from washed-out pitch attitude.

\section{Simulation Facility and Test Procedure}

The piloted simulation was conducted on the Ames Research Center six-degree-of-freedom Vertical Motion Simulator (VMS). The VMS provides extensive cockpit motion for use in studying the handling qualities of and advanced guidance concepts for existing and proposed aircraft 15 . The cockpit arrangement is shown in Figure (4) with the pilot, HMD, and head down moving map display. The cockpit was configured with conventional cyclic, collective, and pedal controls. The visual system, an Evans \& Sutherland CT5A, consists of a threewindow display of computer-generated imagery (CGI). A contour map of the CGI data base used for the simulation is shown in Figure (5), with contour lines scaled by $10 \mathrm{ft}$. The data base was derived from the DMA terrain data from the intended flight test area, near Carlisle, Pennsylvania. The area is $77^{\circ} 30^{\prime}$ to $77^{\circ} 10^{\prime}$ West longitude by $39^{\circ} 59^{\prime}$ to $40^{\circ} 15^{\prime}$ North latitude, and is considered fairly rugged. A series of waypoints are shown positioned on the map connected by straight lines, and used as the mission course for the trajectorygeneration algorithm to follow. Additionally the database was seeded with trees up to $100 \mathrm{ft}$ high and 40 $\mathrm{ft}$ wide. These obstacles were not available to the dynapath algorithm for trajectory calculation.

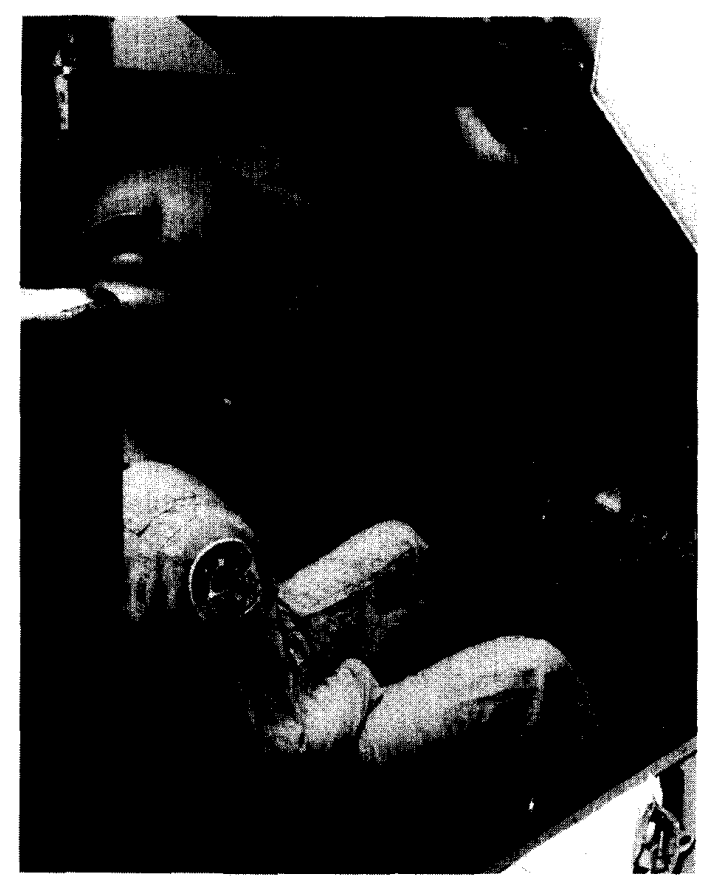

Figure 4. Pilot and Cockpit configuration. 


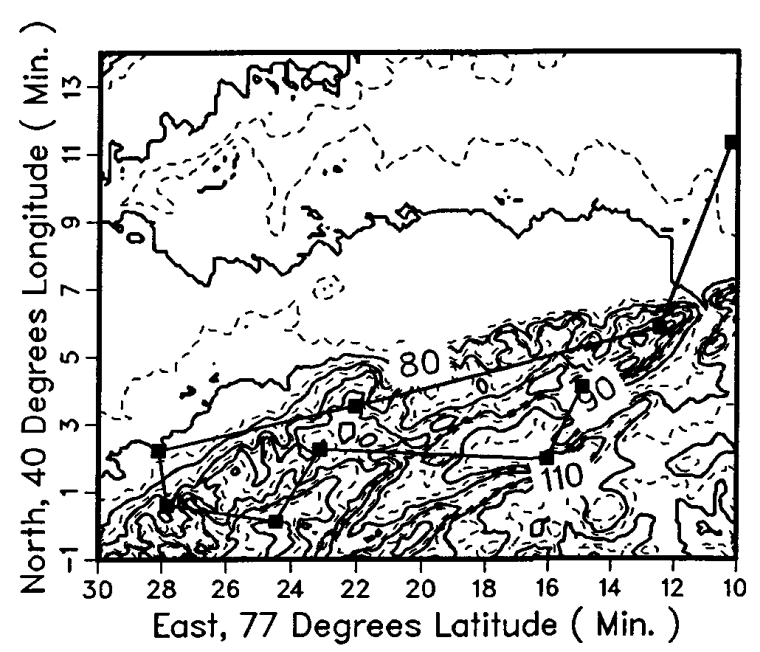

Figure 5. Mission area contour map.

The simulated helicopter was a UH-60A.16 The math model is a nonlinear, blade-element ten-degree-offreedom representation of the aircraft. The degrees of freedom are six rigid-body, rotor flapping and lagging, air mass, and hub rotational. The UH-60A flight-path stabilization (FPS), digital stability augmentation system (SAS), and analog SAS are also included in the model. The pilots typically used the FPS flight control system throughout the simulation. The aircraft navigation/sensor suite was modeled as an integrated Global Positioning System (GPS) and Inertial Navigation Unit (INU). The actual UH-60 STAR flight research computers hosted the trajectory-generation algorithm, trajectory coupler, HMD guidance and display software. The flight computers were integrated into the VMS facility to emulate the flight test systems architecture.
Eighteen helicopter pilots representing NASA, the U.S. Army and the U.S. Air Force and the avionics and airframe industry participated in the evaluations. A total of 287 simulation runs were conducted by the primary NASA, and U.S. Army project pilots. The test matrix is shown in Table (1).

The evaluations consisted of a baseline case ( 80 knots airspeed, $25^{\circ}$ maximum bank command, 4 sec lead aircraft time-constant, 7 pathway lines, $150 \mathrm{ft}$ set clearance above-ground-level (AGL), no target identification task, no turbulence, and $10 \mathrm{mi}$ visibility), and the fourteen variations shown in Table (1). The variations included airspeed (60 and 100 knots), increased maximum bank command $\left(35^{\circ}\right)$, lead aircraft time-constant ( 3 and $5 \mathrm{sec}$ ), number of pathway lines (10 and 20), different set clearance altitudes (100 and $200 \mathrm{ft} A G L$ ), a target identification task, inclusion of moderate turbulence, and reduction of visibility to $1 / 4$ and $1 / 2$ mile. The pilots started each run with the trajectory guidance information displayed on the HMD and with the helicopter trimmed at the correct altitude, heading and airspeed for the commanded trajectory.

The pilots were given three types of tasks. In the case of $200 \mathrm{ft}$ and $150 \mathrm{ft}$ AGL set clearance, the task was to precisely follow the flight-path vector/predictor and phantom aircraft guidance to determine flight technical error. In the case of the $100 \mathrm{ft}$ AGL set clearance, the pilot was asked to use the guidance for general navigation but to override the command as required for obstacle avoidance. The final task was to again use the guidance for general navigation but his primary task was to locate and identify targets. The motivation behind the location and identification task was to investigate pilot situational awareness while flying the system. Ground and air vehicles were placed pseudo-randomly throughout the run, either on the simulated terrain or at the set clearance altitude (150 ft AGL). Ten targets appeared randomly throughout a $20 \mathrm{~min}$ run constrained by the fact the targets were to appear one at a time and would remain on for a maximum of $30 \mathrm{sec}$.

Table 1. Simulation test matrix

\begin{tabular}{ccccccccc}
\hline $\begin{array}{c}\text { Test } \\
\text { Condition }\end{array}$ & $\begin{array}{c}\text { Airspeed } \\
\text { (knots) }\end{array}$ & $\begin{array}{c}\text { Max. bank } \\
\text { command } \\
\text { (deg) }\end{array}$ & $\begin{array}{c}\text { Lead } \\
\text { aircraft } \\
\text { time }(\mathrm{sec})\end{array}$ & $\begin{array}{c}\text { Pathway } \\
\text { lines }\end{array}$ & $\begin{array}{c}\text { Set-clear } \\
\text { altitude } \\
\text { (ft, }\end{array}$ AGL) & $\begin{array}{c}\text { Target } \\
\text { ident. }\end{array}$ & $\begin{array}{c}\text { Turbulence Visibility } \\
\text { (moderate) }\end{array}$ & $\begin{array}{c}\text { VGiles) } \\
\text { (miles }\end{array}$ \\
\hline 1 & 80 & 25 & 4 & 7 & 150 & OFF & OFF & 10 \\
2 & 60 & 25 & 4 & 7 & 150 & OFF & OFF & 10 \\
3 & 100 & 25 & 4 & 7 & 150 & OFF & OFF & 10 \\
4 & 80 & 35 & 4 & 7 & 150 & OFF & OFF & 10 \\
5 & 80 & 25 & 3 & 7 & 150 & OFF & OFF & 10 \\
6 & 80 & 25 & 5 & 7 & 150 & OFF & OFF & 10 \\
7 & 80 & 25 & 4 & 10 & 150 & OFF & OFF & 10 \\
8 & 80 & 25 & 4 & 20 & 150 & OFF & OFF & 10 \\
9 & 80 & 25 & 4 & 7 & 100 & OFF & OFF & 10 \\
10 & 80 & 25 & 4 & 7 & 200 & OFF & OFF & 10 \\
11 & 80 & 25 & 4 & 7 & 150 & ON & OFF & 10 \\
12 & 80 & 25 & 4 & 7 & 150 & OFF & ON & 10 \\
13 & 80 & 25 & 4 & 7 & 150 & OFF & OFF & $1 / 4$ \\
14 & 80 & 25 & 4 & 7 & 150 & OFF & OFF & $1 / 2$ \\
\hline \hline
\end{tabular}


The targets were randomly placed within $\pm 15^{\circ}$ of the aircraft's heading and at a range of 1 to $2 \mathrm{~km}$ in front of the aircraft. Once a target was located and identified the pilot engaged one of two cyclic buttons to classify the targets as either "friend or foe". The friend targets were an $\mathrm{AH}-64$ helicopter and an $\mathrm{M}-1$ tank, the foes were a Mi-24 helicopter and a Zsu-23 rocket launcher. Feedback for correct target identification was shown by exploding the targets; incorrect identification by pitching the target up at $90^{\circ}$.

At the conclusion of each of the runs tracking performance and target identification accuracy were assessed. The NASA research pilots were also asked to give a Cooper Harper $(\mathrm{CH})$ pilot rating upon conclusion of each run type. The $\mathrm{CH}$ rating scale, simply stated, is a scale from 1 to 10 where 1 is the best and 10 the worst. 17 The rating is based upon the pilots ability to obtain a desired level of performance and the workload required to maintain that level. Desired performance $(\mathrm{CH} 1$ to 3.5$)$ for the tracking task was to maintain $\pm 25 \mathrm{ft} 1-\sigma$ (standard deviation) vertically and $\pm 50 \mathrm{ft} 1-\sigma$ laterally throughout the run. For the general navigation/ obstacle avoidance run the desired performance was to miss all the trees and maintain \pm 50 $\mathrm{ft} 1-\sigma$ vertically and $\pm 100 \mathrm{tt} 1-\sigma$ laterally. And finally, the desired performance for the general navigation/target identification run was to be able to use the guidance system to track the waypoint course and to achieve $80 \%$ accuracy target location and identification. With all the tasks, it was defined as unacceptable for the pilot to hit a tree or the ground.

\section{Results and Discussion}

\section{Performance Results}

For all the test combinations listed in Table (1) the pilots were able to, on average, maintain the desired level of performance using the flight-path vector/predictor, phantom aircraft, and pathway display symbols. An example ground track for the simulation runs is shown in Figure (6) superimposed on the contour map described earlier. A plot of the vertical trajectory and terrain profile for the example run is shown as a function of distance traveled in Figure (7). These plots are shown as representative of the lateral and vertical maneuvering commanded by the trajectory-generation algorithm, and flown by the pilots for the test configurations.

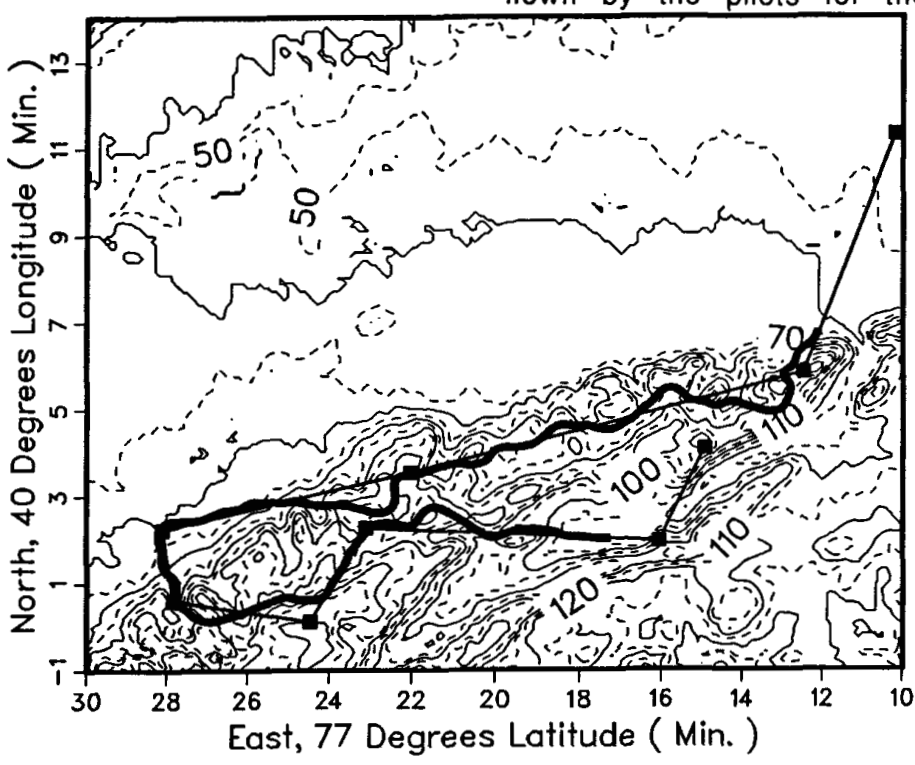

Figure 6. Example ground track

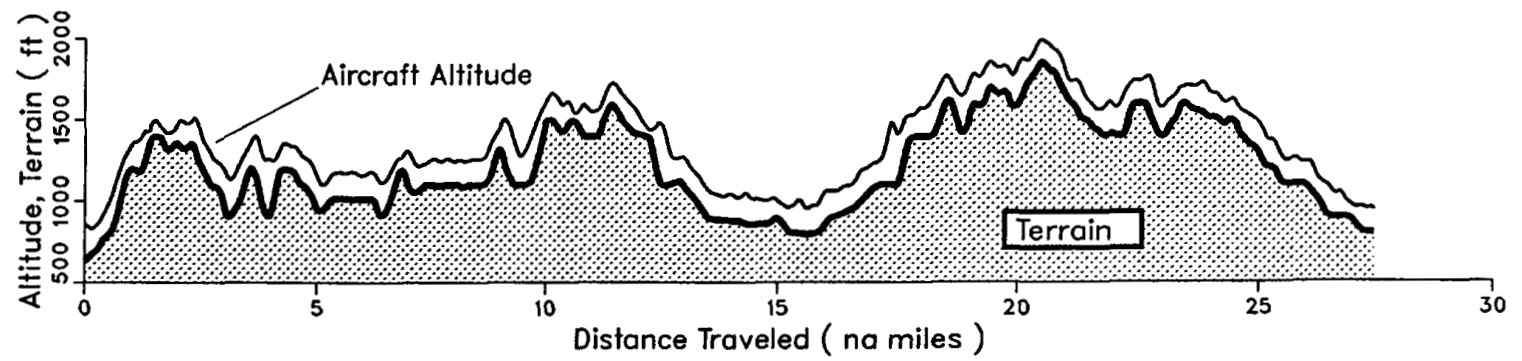

Figure 7. Example vertical trajectory and terrain profile 
The lateral, vertical, and terrain tracking performance as well as the average $\mathrm{CH}$ rating for all configurations are summarized in Figure (8). The Figure shows the mean and $1-\sigma$ tracking performance for each configuration tested. The lateral and vertical pilot tracking plots quantify the pilots ability to track the commanded trajectory using the HMD guidance and display symbols. The terrain tracking graphs shows the variance in altitude above the terrain for the configuration tested. As evident, with exception of the $100 \mathrm{ft}$ AGL set clearance case (condition 9), and the target identification case (condition 9) the lateral and vertical tracking performance was under $\pm 30 \mathrm{ft}$ and $\pm 15 \mathrm{ft}$ respectively, well within the desired performance described earlier.

As discussed earlier, the pilots task when flying the low set clearance altitude was somewhat different than the other cases. For the low set clearance altitude (100 ft $A G L$ ), the pilot was flying at the altitude of trees within the database which are not included in the trajectorygeneration algorithm. For this reason the pilots were instructed to only use the guidance as an aid and to manually avoid obstacles that were in the displayed trajectory. All the pilots successfully avoided the obstacles within the database, and were able to track the guidance within the desired performance described for this case. It is interesting to note that the pilots primarily chose lateral maneuvering to avoid the obstacles. This is evident by comparing the lateral deviation from the commanded path $(\sim 60 \mathrm{ft} 1-\sigma)$ with the lateral tracking error measured in the baseline case $(\sim 15 \mathrm{ft} 1-\sigma)$. This is due to the pilot initiating maneuvers around obstacles and then tracking back to the commanded path. The vertical tracking performance (15 $\mathrm{ft} 1-\sigma$ ) is very similar to the baseline configuration (10 ft $1-\sigma)$, thus implying lateral maneuvering was the first choice among the pilots for manual obstacle avoidance. For the target identification case the lateral and vertical tracking performance was similar to the baseline configuration while the pilots tracked the guidance and scanned the horizon for targets. But typically the pilots would pull themselves from the desired course to pursue and identify targets then reengage the guidance system to continue the mission. Thus the lateral and vertical pilot tracking performance data shows the results of these maneuvers. All the pilots were able to complete the mission with an average $83 \%$ accuracy target location and identification in an average response time of 18.6 seconds.

\section{Pilot Evaluations}

As mentioned earlier, $\mathrm{CH}$ pilot-rating data were collected during the evaluation. The baseline configuration had an average rating of 3 , indicating that desired performance was achievable with minimal pilot effort. The slow speed ( $60 \mathrm{knot}$ ) case was given a rating of 4.5 indicating that desired performance required moderate pilot compensation. The increase in pilot workload from the baseline is attributed to two factors. First, the trajectory-generation algorithm calculates the lateral maneuvers based upon path curvature derived from the helicopters current speed and the bank angle control described earlier, thus with the lower speed the trajectory will have higher turn rates. The second factor, and the one the pilots felt was more dominant, is that at 60 knots the UH-60 stabilator becomes more active and also the FPS flight control system switches between turn-coordination and heading hold modes causing a degradation in the helicopter's basic handling qualities. The high speed (100 knots) case was rated as 3.25 , almost the same as the baseline with possibly a slightly higher pilot effort. The steep maximum commanded bank $\left(35^{\circ}\right)$ case was rated as a 4 again due to the higher turn rates.

The reduction in the lead-aircraft and flight-path vector/predictor time-constant to $3 \mathrm{sec}$, case 5 , effectively forces the pilot to track tighter with the guidance symbology. This is evident by the $50 \%$ reduction in lateral tracking error shown for this configuration. But the pilot also has to work harder to position the flight-path vector/predictor on the leadaircraft. Thus the increase in the average $\mathrm{CH}$ rating to a 4.25. The increase in the lead-aircraft and flight-path vector/predictor time-constant to $5 \mathrm{sec}$, case 6 , allows looser tracking with the guidance symbology. This is shown by the slightly larger statistical variation in the tracking results than the baseline. The rating for this case was a 4, and when the pilots were asked why this case was rated lower than the baseline, they indicated that the guidance seemed to make them sloppy in their tracking. Another display parameter evaluated was the number of pathway lines presented, cases 7 and 8 . For these 10 and 20 line pathway configurations the ratings were 3.5 and 4.5 respectively. The primary reason was the corresponding increase in display clutter with little if any increase in useful information. The pilots did indicate that the pathway with seven lines did give very useful information regarding the trend of the Dynapath trajectory.

The low-altitude (100 ft AGL set clearance) case, as discussed before, had the added pilot obstacleavoidance override task. The pilots gave this case an average $\mathrm{CH}$ rating of 4 based upon the moderate increase in pilot compensation for this particular task. The high altitude (200 ft AGL set clearance) case was rated as a 3.25 , again very similar to the baseline.

The general navigation/target location and identification task, case 11, was rated as a 3 . As the performance data shows, the pilots were able to satisfactorily complete the described task. All the eighteen evaluation and guest pilots that flew the simulation felt that this task was very representative of the tasks to be accomplished in an operational scenario. This task highlighted the fact that the pilot could use the guidance and display system without any loss of situational awareness of the outside world.

The pilots also flew the system with adverse weather effects, i.e. turbulence and limited visibility. The major effect from the turbulence was the extra head vibration which caused a slight display jitter with the body and inertially referenced symbols. The pilots gave this 

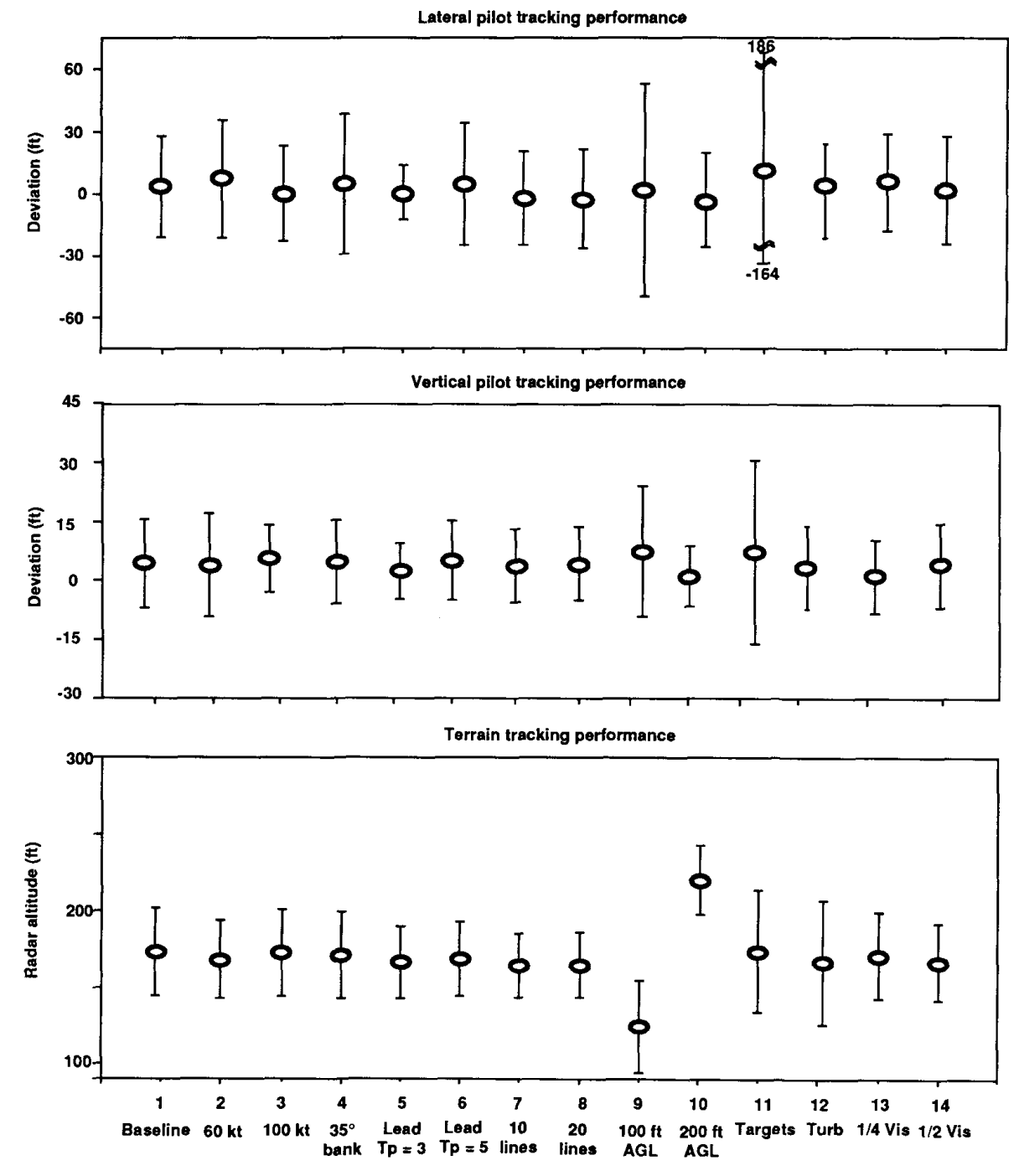

\begin{tabular}{|l|l|l|l|l|l|l|l|l|l|l|l|l|l|l|}
\hline $\begin{array}{l}\text { Ave CH } \\
\text { pilot } \\
\text { rating }\end{array}$ & 3 & 4.5 & 3.25 & 4 & 4.25 & 4 & 3.5 & 4.25 & 4 & 3.25 & 3 & 4.5 & NR & 3.5 \\
\hline
\end{tabular}

Figure 8. Pilot performance and evaluations 
configuration a rating of 4.5 due to the increased compensation and extra visual fatigue caused by the jitter. The reduced visibility runs $(1 / 4$ and $1 / 2 \mathrm{mi})$ were also done. The pilots rated the $1 / 2 \mathrm{mi}$ run as a 3.5 , a slight increase in workload compared to the baseline. The $1 / 4 \mathrm{mi}$ was not rated because the pilots did not feel the simulated visual scene adequately represented actual $1 / 4$ mi visibility.

\section{Conclusions}

A low-altitude, maneuvering penetration guidance algorithm for helicopter operations has been developed and evaluated in a full-motion simulator. The evaluation pilots were able to manually track the HMD guidance through various combinations of terrain, speeds, and weather representative of system use. The guidance can be followed with low pilot workload without detracting from his awareness of the outside world. The pilot is able to combine the guidance with his visual senses to optimize the mission success in varying weather/threat conditions. The computer-aiding system has matured through the extensive use of piloted simulation, and integration of the concept into the UH-60 STAR helicopter is proceeding towards a flight test early in 1992.

\section{Acknowledgements}

The authors are grateful to George Tucker, Bill Hindson, and Maj. Loran Haworth (Ames Research Center), and Tom Davis, Gary Amatrudo, Cpt. Ray Jones, CW4 Brad Powell, and Maj. Paul Losier (U.S. Army), Lt. Col Gary Brovetto (U.S. Air Force), and Nick Lappos (Sikorsky Aircraft) for their participation as evaluation pilots.

\section{References}

1. Tripp, E.: "SOF Helicopters: Operating on the Dark Side. Rotor \& Wing International, Dec. 1987.

2. Wendl, M.J.; Katt, D.R.; and Young, G.D.: Advanced Automatic "Terrain Following/Terrain Avoidance Control Concepts Study. 1982 NAECON Conference Proceedings.

3. Russ, D.E. ; Houtz, J.E.; Rothstein, S.W.: Evaluations of Alternative Terrain Following/Terrain Avoidance (TF/TA) Systems Designs Using Pilot in the Loop Simulation. AGARD CP-387, Oct. 1985.

4. Denton,R.V.; Jones, J.; and Froeberg, P.L. : A New Technique for Terrain Following/Terrain Avoidance Guidance Command Generation. AGARD CP-387, Oct. 1985.

5. Nordmeyer, R.: Enhanced Terrain Masked Penetration Final Technical Report. AFWAL TR86-1079, Sept. 1986.

6. Dorr,D.W. : Rotary Wing Aircraft Terrain Following/Terrain Avoidance System Development. NASA TM 88322, 1986.
7. Hoffman, J.D.: Terrain Following/Terrain Avoidance/Threat Avoidance for Helicopter Applications. AHS Proceedings National Specialists' Meeting in Rotorcraft Flight Controls and Avionics, Oct. 1987.

8. Pekelsma, N.J.; and Denton, R.V.: Pilot Oriented Aids for Helicopter Automatic Nap of the Earth Flight. AHS Proceedings National Specialists' Meeting in Rotorcraft Flight Controls and Avionics, Oct. 1987.

9. Swenson, H.N.; Hardy, G.H.; and Morris, P.M.: Simulation Evaluation of Helicopter Terrain Following/Terrain Avoidance Concepts. AIAA-8839224-CP AIAA/AHS/ASEE Aircraft Design Systems and Operations Meeting, Oct. 88.

10. Swenson, H.N.: Computer Aiding for Low Altitude Helicopter Flight. NASA TM 103861, May, 1991.

11. Denton, R.V.; Froeberg, P.L.; Jones, J.E.; and Nikoukhah, R.: Terrain Following/Terrain Avoidance Algorithm Study. AFWAL TR-85-3007, May 1985.

12. Denton, R.V.; Pekelsma, N.J.; and Smith, F.W.: Autonomous Flight and Remote Site Landing Guidance Research for Helicopters. NASA CR177478,1987

13. Pekelsma, N.J.; and Smith, F.W.: Optimal Guidance with Obstacle Avoidance for Nap-of-the-Earth Flight. NASA CR-177515, December 1988.

14. Bray, R.; and Scott, B.: A Head Up Display for Low Visibility Approach and Landing. AIAA paper 81 $0130,1981$.

15. Jones, A. D.: Operations Manual: Vertical Motion Simulator (VMS) S.08. NASA TM-81180, 1980.

16. Howlett, J.J: UH-60A BLACKHAWK Engineering Simulation Program: Volume I - Mathematical Model. NASA CR 199309, 1981

17. Cooper, G.E.; and Harper, R.P.: The Use of Pilot Ratings in the Evaluation of Aircraft Handling Qualities. NASA TN D 5113, 1969. 